\title{
gp130 is important for the normal morphogenesis of Meckel's cartilage and subsequent mandibular development
}

\author{
Jung Won Choi ${ }^{1}$, Jung Tak Kim ${ }^{1}$, \\ Jae Han Park', Eui Kyun Park', \\ Sin-Yoon $\mathrm{Kim}^{2}$, Tae-Geon Kwon ${ }^{3}$, \\ Eun-Cheol $\mathrm{Kim}^{4}$ and Hong-In Shin ${ }^{1,5}$
}

${ }^{1}$ Department of Oral Pathology

School of Dentistry, Kyungpook National University

Daegu 700-412, Korea

${ }^{2}$ Skeletal Diseases Genomic Research Center

Kyungpook National University Hospital

${ }^{3}$ Department of Maxillofacial Surgery

School of Dentistry, Kyungpook National University

Daegu 700-412, Korea

${ }^{4}$ Department of Maxillofacial Pathology

Dental College, Wonkwang University

Iksan 570-749, Korea

${ }^{5}$ Corresponding author: Tel, 82-53-420-4994;

Fax, 82-53-427-4918; E-mail, hishin@knu.ac.kr

Accepted 13 March 2007

Abbreviations: BMP, bone morphogenetic protein; CNTF, ciliary neurotrophic factor; CT-1, cardiotrophin-1; gp130, glycoprotein 130; HGF, hepatocyte growth factor; LIF, leukemia inhibit factor; MMP, matrix metalloproteinase; OSM, oncostatin M; PTH, parathyroid hormone; PTHrP, parathyroid hormone related protein; TRAP, tartrate-resistant acid phosphatase

\footnotetext{
Abstract

gp130-mediated signaling is involved in both chondrogenesis and osteogenesis, but its direct role in the formation of embryonic Meckel's cartilage and associated mandibular development has not yet been elucidated. In this study, we examined the influence of gp130 ablation on the developing mandibular Meckel's cartilage by evaluating the morphological and histological changes as well as the gene expression patterns in developing embryonic gp130-/- mice. The ablation of the gp130 gene showed no change in region-specific collagen mRNA expression except for a slight delay in its expression but caused shortened embryonic Meckel's cartilage, delayed hypertrophic chondrocyte maturation and subsequent bony replacement with characteristic bending of the intramandibular Meckel's cartilage. The bending of Meckel's cartilage led to a narrow mandibular arch at the rostral area with poor cortical plate formation. These findings indicate that gp130-mediated signaling is important for the normal morphogenesis of Meckel's cartilage and subsequent mandibular development.
}

Keywords: cartilage; cytokine receptor gp130; chondrogenesis; in situ hybridization; mandible; morphogenesis

\section{Introduction}

The various histological morphogenetic events that occur during embryonic mandibular development depend on the proper formation of Meckel's cartilage, which serves as the primordium of the mandible and guides its early morphogenesis. A derivative of the cranial crest, Meckel's cartilage is regarded as the prominent first skeletal component of the developing mandible (Tomo et al., 1997; Ishizeki et al., 2001). A variety of growth factors, such as EGF (Shum et al., 1993), fibroblast growth factor (FGF) (Wilson and Tucker, 2004), TGF (Ito et al., 2002) and hepatocyte growth factor (HGF) (Amano et al., 1999), and a complicated BMP-Msx-FGF signaling network participate in the initiation and maintenance of chondrogenesis of Meckel's cartilage and osteogenesis of the mandible (Macdonald and Hall, 2001; Wilson and Tucker, 2004). Parathyroid hormone related protein (PTHrP), which shares a receptor with parathyroid hormone (PTH), constitutes a signaling pathway that negatively regulates the rate of chondrocyte differentiation in endochondral bone formation during embryogenesis (Yamazaki et al., 1997; Long and Linsenmayer, 1998). Numerous investigators have suggested that parathyroid hormone (PTH) and parathyroid hormone related protein (PTHrP) play a role in skeletal homeostasis, in part by regulating gp130-associated cytokines (Hetnabb and Rousselle, 2000).

gp130-associated cytokines such as IL-6, IL-11, leukemia inhibit factor (LIF), oncostatin M (OSM), ciliary neurotrophic factor (CNTF), and cardiotrophin-1 (CT-1) share the ubiquitously expressed common signal transducing receptor component gp130 and make up a cytokine family based on their common structure and function. These cytokines bind with their cognate receptors and then form a receptor complex by inducing homodimerization or heterodimerization of gp130 with LIF receptor or OSM receptor for signal transduction. The gp130-mediated signaling triggered by gp130-associated cytokines induces diverse biologic functions in immune, hematopoietic, and nervous systems as well as in bone (Taga and Kishimoto, 1997; Betz et al., 1998; Bravo and Heath, 2000). 
gp130 regulates bone turnover and bone size by at least two distinct intracellular pathways, STAT1/3 and SHP2/ras/MAPK, which are selectively activated in osteoblasts (Sims et al., 2004). The gp130-/- mice had shortened limbs, as also observed in PTHrP-/- and PTH/PTHrP receptor-/mice (Lanske et al., 1999), suggesting impairment not only in osteoblast differentiation but also in chondrocyte differentiation. As in other skeletal elements, Meckel's cartilage formation is initiated by condensation of prechondrogenic cells, which differentiate into chondrocytes and give rise to characteristic rod-shaped cartilage. Meckel's cartilage serves as the primordium of the mandible, malleus, incus, and the sphenomandibular ligament, depending on the distinct subdivisions of chondrocytes (Miyake et al., 1996; Harada and Ishizeki, 1998). The rostral and auricular ends of Meckel's cartilage support the mandibular development and give rise to the inner-ear ossicles, the malleus, and the incus, respectively, through endochondral-type ossification. In contrast, the midportion of Meckel's cartilage transforms into noncartilagenous tissue and forms the sphenomandibular ligament soon after birth (Yamazaki et al., 1997; Ishizeki et al., 2001). However, its direct role in embryonic mandibular development has not yet been well elucidated.

In this study, we examined the influence of gp130-mediated signaling on developing mandibular Meckel's cartilage by evaluating the morphological and histological changes as well as the gene expression patterns in the developing embryonic gp130-/- mandible.

\section{Materials and Methods}

\section{Animals}

gp130-deficient embryos were obtained by crossing heterozygous mice carrying a disrupted gp130 gene through homologous recombination with a targeting vector that inserted a pMCINeo-poly $(A)$ cassette into the Hindlll site in exon 2 just downstream of the translation initiation codon of the gp130 gene (Yoshida et al., 1996). Mice were genotyped by PCR using genomic DNA isolated from tail clips. The embryos were obtained before birth by cesarean section at different days of gestation after timed mating. These studies were approved by the institutional animal care committee of School of Dentistry, Kyungpook National University. All mice were provided a standard chow diet and water.

\section{Skeletal preparation}

Three to four embryonic wild and gp130-deficient mice obtained at each developing stage of E15.5 to E18.5 by cesarean section were eviscerated and fixed in $95 \%$ ethanol for $24 \mathrm{~h}$ and stained for 10 days in a staining solution consisting of $1 \mathrm{vol}$ of $0.1 \%$ Alizarin red S (Sigma Chemical Company, St. Louis, MO) in $95 \%$ ethanol, 1 vol of $0.3 \%$ Alcian blue 8GX (Sigma Chemical Company, St. Louis, $\mathrm{MO}$ ) in $70 \%$ ethanol, and $1 \mathrm{vol}$ of acetic acid, and $17 \mathrm{vol}$ of absolute ethanol (Nifuji et al., 1997). The stained animals were rinsed with $100 \%$ ethanol and kept in $20 \%$ glycerol $/ 1 \% \mathrm{KOH}$ at $37^{\circ} \mathrm{C}$ for $16 \mathrm{~h}$. The specimens were then kept at room temperature until the skeletons became clearly visible. Photographs were taken using a stereoscopic microscope (Olympus, Japan).

\section{Histologic preparation}

Dissected heads including the mandible from four embryos of each wild type and gp130-deficient at E15.5-E18.5 were fixed in 10\% neutral formalin for $24 \mathrm{~h}$ at $4^{\circ} \mathrm{C}$. After washing with PBS, they were then dehydrated in an ascending graded ethanol series, cleared with xylene, and embedded in paraffin. Serial sections of $5-\mu \mathrm{m}$ thickness were cut from each block at the horizontal plane and mounted on silane-coated glass slides.

\section{Double staining for von Kossa and tartrate-resistant acid phosphatase (TRAP)}

To detect the resorptive activity of the calcified matrix of osteoclastic cells, the sections were subjected to double staining with von Kossa and TRAP staining. First, the deparaffinized sections were incubated with $5 \%$ silver nitrate for $30 \mathrm{~min}$, exposed to daylight for $15 \mathrm{~min}$, and then incubated with $5 \%$ sodium thiosulfate for 2 min. After von Kossa staining, the sections were incubated for $10 \mathrm{~min}$ with a mixture of $0.1 \mathrm{mg} / \mathrm{ml}$ naphthol AS-MX (Sigma), $0.5 \% \mathrm{~N}, \mathrm{~N}$-dimethylformamide, and $0.6 \mathrm{mg} / \mathrm{ml}$ fast red violet salt (Sigma) in $0.1 \mathrm{M}$ acetate buffer solution $\left(\mathrm{pH} \mathrm{5.0)}\right.$ at $37^{\circ} \mathrm{C}$ to detect osteoclastic cells showing TRAP enzymatic activity (Shin et al., 2004).

\section{In situ hybridization}

Digoxigenin-labeled single strand RNA probes for type I, II, and X collagen were prepared using a DIG RNA labeling kit (Roche, Germany) according to the manufacturer's instructions (Nicole et al., 1998). Deparaffinized sections were pretreated with Proteinase $\mathrm{K}$ in TE $(0.1 \mathrm{M}$ Tris- $\mathrm{HCl} \mathrm{pH} 8.0,0.5 \mathrm{M}$ EDTA pH 8.0) for $12 \mathrm{~min}$ at $37^{\circ} \mathrm{C}$, and immediately post-fixed in $4 \%$ paraformaldehyde in $0.1 \mathrm{M}$ phosphate buffer for $10 \mathrm{~min}$. Sections were then treated 
with $0.2 \mathrm{~N} \mathrm{HCl}$ to inactivate endogenous alkaline phosphatase and acetylated with $0.25 \%$ acetic anhydride in $0.1 \mathrm{M}$ triethanolamine $(\mathrm{pH} 8.0)$ for 10 min. Sections were hybridized with digoxigenin-labeled antisense probes at $50^{\circ} \mathrm{C}$ for $16 \mathrm{~h}$ in a moisture chamber. After hybridization, slides were washed briefly with $5 \times$ SSC containing $50 \%$ formamide, twice with $2 \times \mathrm{SSC}$ for $30 \mathrm{~min} /$ wash, and twice with $0.2 \times \mathrm{SSC}$ for $20 \mathrm{~min} /$ wash at $50^{\circ} \mathrm{C}$, respectively. The hybridized digoxigenin-labeled probe was detected using a nucleic acid detection kit (Roche) according to the manufacturer's instruction. After color reaction, the slides were rinsed with water, counter stained with $1 \%$ methyl green, and mounted.

\section{Histomorphometry}

The measurements of tissue sizes were made from digital images of mandibles dissected from wild type and gp130-/- fetuses processed for skeletal preparation at E15.5-E18.5 using the iMT system (Daegeon, Korea). For the statistical analysis total length of mandible (from symphysis to condylar process), Meckel's cartilage (from rostal end to auricular end), and replaced Meckel's cartilage area by bone at hypertrophied cartilage zone were measured 3 times. The experimental data are expressed as mean \pm S.E. The significance of differences was analyzed by Tukey's multiple range tests after ANOVA. $P<0.05$ was conventionally considered statistically significant.

\section{Results}

\section{Morphology of the developing embryonic mandible and associated Meckel's cartilage}

gp130-/- fetuses developed to term but died within 1 day after birth for unknown reasons as previously reported. On gross examination, the fetuses appeared normal, except that they were generally smaller and had shorter limbs than those of heterozygous and wild type littermates.
The prepared skeletal samples from gp130-/- embryos from E15.5 to E18.5 had smaller mandibles and the replacement of intramandibular Meckel's cartilage with mineralized bony tissue was delayed and reduced as compared to age-matched wild type embryos (Figure 1). The incremental growth of Meckel's cartilage and mandible was significantly retarded statistically by ablation of gp130 gene during embryonic development at E15.5-E18.5 with significant narrowing of hypertrophic chondrocytic maturation zone (Figure 2). The Meckel's cartilage in developing embryonic mandibles was partially surrounded by a mandibular plate extending medially from the superior and inferior borders. Meckel's cartilage was noted as blue colored, relatively straight rods with vertical angulation at the auricular ends, which formed the primordium of the malleus and incus. Meckel's cartilage fused with the rostrum and formed a v-shaped frame at the medial side of the developing mandible (Figure $3 \mathrm{~A}$ ). The replacement of Meckel's cartilage with mineralized bony tissue at the anterior portion was definitely noted from E16.5 in wild type embryos (Figure 1B) and from E17.5 in gp130-/- embryos (Figure 1G), suggesting delayed maturation of chondrocytes destined to become hypertrophic chondrocytes. The Alcian blue-stained intramandibular Meckel's cartilage tissue was gradually integrated into the Alizarin red-stained mandibular bone below the mental foramen region. The bony replacement extended to the molar tooth area with time, and approximately $40 \%$ of the intramandibular Meckel's cartilage was replaced by Alizarin red-stained bony tissue at E18.5 in both wild type and gp130-/- littermates (Figure 1D, 1H). Strikingly, the gp130-/- developing embryonic mandibles had a narrower mandibular arch particularly in the rostral area, and bending of intramandibular Meckel's cartilage was clearly noted from E17.5 (Figure 3B). These findings indicate that gp130-mediated signaling might be important for normal morphogenesis of Meckel's cartilage and subsequent mandibular development.
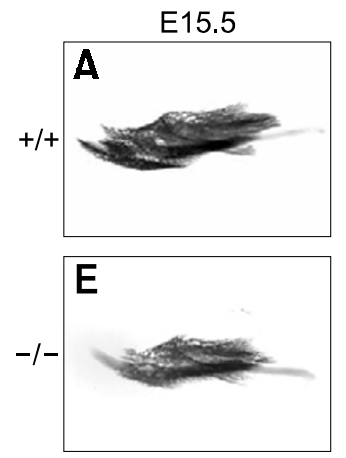
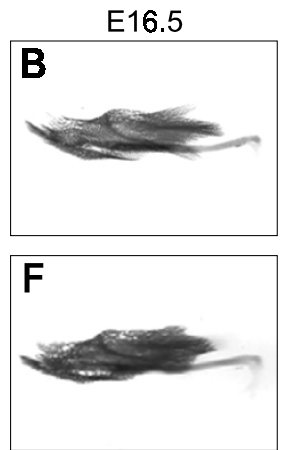

E17.5
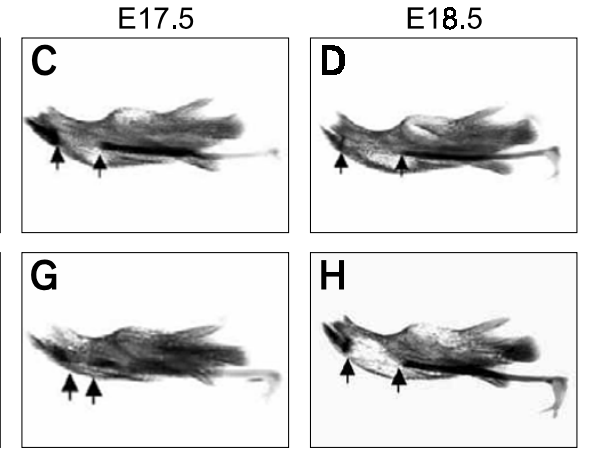

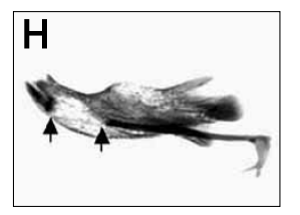

Figure 1. Skeletal preparation of developing mandibles from E15.5 to E18.5 wild type and gp130-/embryos. gp130-/- mandibles were smaller and the replacement of intramandibular Meckel's cartilage (arrows) with mineralized bony tissue was delayed and reduced compared to age-matched wild type mandible (original magnification $\times 20$ ). 
A

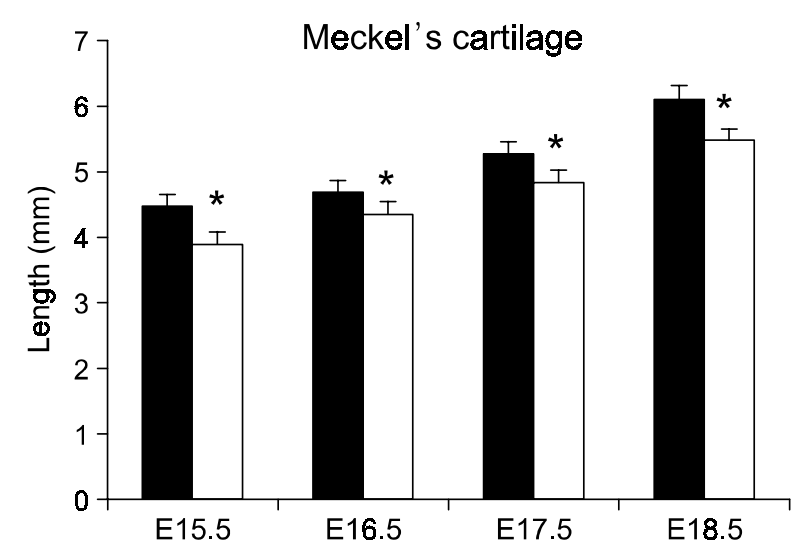

B

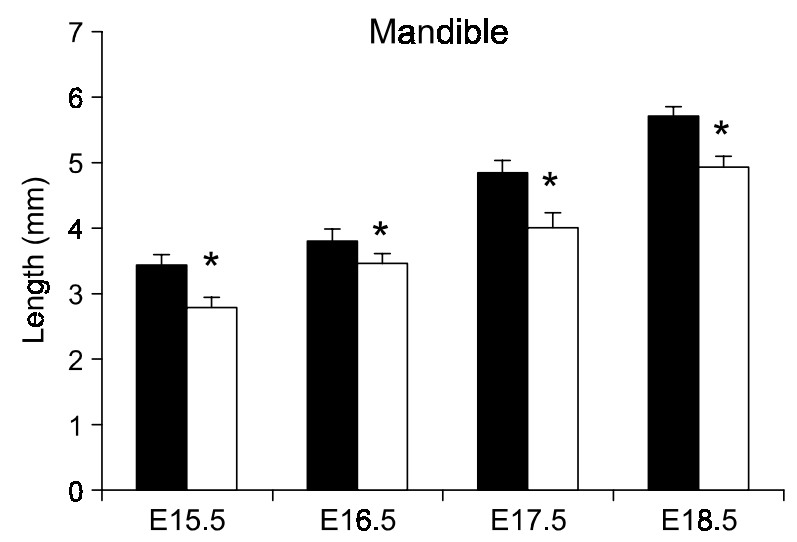

C

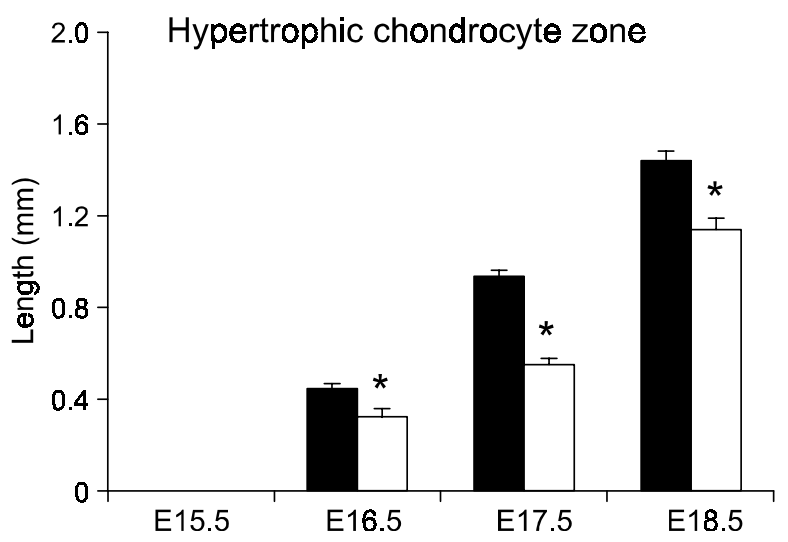

Figure 2. Incremental growth of Meckel's cartilage (A), mandible (B), and hyeprtrophic chondrocyte zone of developing Meckel's cartilage (C) during embryonic stage from E15.5 to E18.5 in wild type (black bar) and gp130 deficient mice (white bar). Data from 3 skeletal preparation samples was represented as mean \pm S.E. ${ }^{*} P<0.05$ vs. wild type.
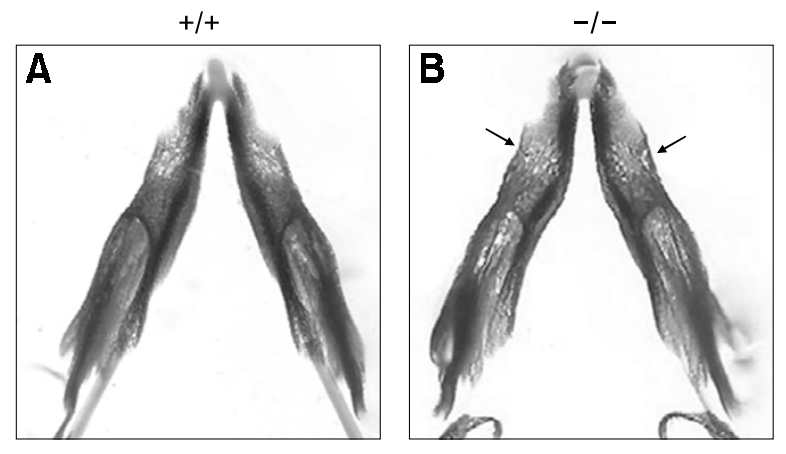

Figure 3. Skeletal preparation of developing mandibles from wild type and gp130-/- embryos at E17.5. The gp130-/- embryonic mandible had a narrow mandibular arch and characteristic bent intramandibular Meckel's cartilage (arrows) undergoing replacement with bone through endochondral ossification (original magnification $\times 40$ ).

\section{Histologic changes in embryonic intramandibular Meckel's cartilage}

The sections double stained for von Kossa and TRAP activity in the gp130-/- developing embryonic mandible showed delayed hypertrophic chondrocyte maturation and replacement of mineralized cartilage matrix with retarded development of Meckel's cartilage and mineralized mandibular bone (Figure 4).

The anterior portion of the wild type intramandibular Meckel's cartilage showed hypertrophic chondrocytic maturation with mineralized bone collar formation adjacent to the developing incisors in E15.5 (Figure 4A), one day earlier than observed in the gp130-/- intramandibular Meckel's cartilage (Figure 4F). With time, the mineralized hypertrophic chondrocyte area extended in an anterior-posterior direction to the molar region, except for the rostrum. A significant portion of Meckel's cartilage surrounded by thin mineralized bone was devoid of hypertrophic chondrocytic maturation and was separated by a thin layer of fibrous connective tissue, unlike the hypertrophic chondrocyte layers that showed no fibrous separation between the bone plate and Meckel's cartilage (Figure 4B, G). The mineralized matrix of the hypertrophic chondrocyte layers was gradually resorbed by TRAP-positive cells in the lateral surface adjacent to the developing incisors, which resulted in saucerization of the lateral surface of Meckel's cartilage (Figure 4 B,C,F,G). The resorbing gp130-/- TRAP positive osteoclastic cells were fewer in number than the wild type osteoclastic cells (Figure 4C), but were characteristically larger and rounder in shape with marked multinucleation (Figure 4G). The resorbing Meckel's cartilage tissue was ultimately replaced by trabecular bone intruding from the mandibular plate that surrounds Meckel's cartilage (Figure 4D, H). 
Unlike the growth palate of long bone, which shows gradual and ordered chondrocyte maturation and replacement of mineralized cartilage with primary spongiosa formation, Meckel's cartilage did not undergo ordered and axial replacement of cartilage tissue into bone, but rather replaced cartilage tissue randomly from the lateral side of developing incisors. At E18.5, a large part of intramandibular Meckel's cartilage disappeared and was integrated into the lingual plate of the mandible (Figure 4D, H), indicating that Meckel's cartilage contributes to thick mandibular lingual plate formation. However, delayed bony integration of hypertrophied chondrocyte layers, resulting in thin and poor cortical mandibular plate formation, was observed in gp130-/Meckel's cartilage with bending of Meckel's carti- lage and developing mandible (Figure 4G, H). These findings suggest that gp130-mediated signaling influences morphogenesis of Meckel's cartilage and subsequent mandibular development.

\section{Collagen gene expression patterns in embryonic Meckel's cartilage}

Expression patterns of collagen type I, II, and X did not differ between wild type and gp130-/- developing Meckel's cartilage (Figures 5-7). At E15.5, the rod-shaped Meckel's cartilage fused at the rostral ends with formation of the symphysis. The Meckel's cartilage was surrounded by perichondrium expressing type I collagen. In addition, the lateral side of the developing mandibular cortical plates of
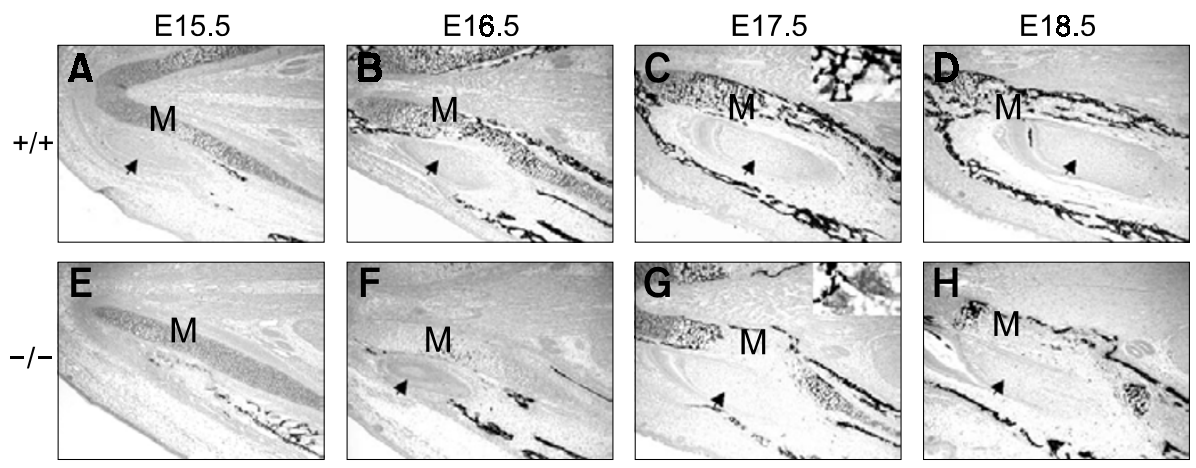

Figure 4. von Kossa and TRAP double staining of the developing mandibles of wild type (A-D) and gp130-/ embryos (E-H) from E15.5 to E18.5. The mineralized tissue stained with von Kossa (stained as black) was reduced in the gp130-/- embryonic mandible $(\mathrm{F}-\mathrm{H})$. The mineralized chondroid matrix of intramandibular Meckel's cartilage (M) adjacent to the incisors (I) was resorbed by TRAP+ multinucleated giant cells. The gp130-/- TRAP+ multinucleated giant cells were characteristically larger and rounder and contained numerous nuclei compared to wild type TRAP+ multinucleated giant cells (inlet). Note the bending of intramendibular Meckel's cartilage and associated lingual plate (arrows) in developing mandible of gp130-/- embryos $(G, H)$. (original magnification $\times 100$, inlet $\times 200$ ).
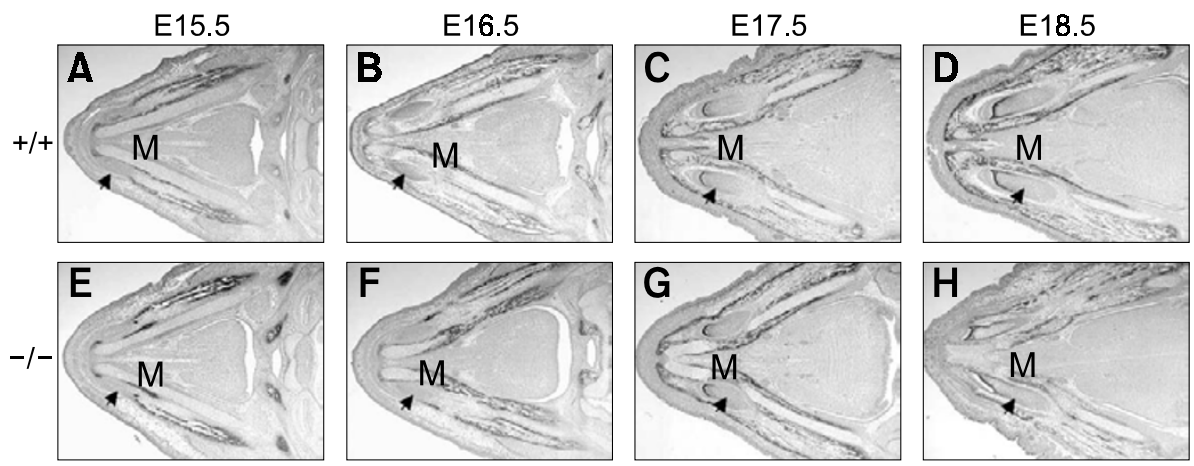

Figure 5. In situ hybridization for collagen type I mRNA in wild type (A-D) and gp130-/- (E-H) developing mandibles from E15.5 to E18.5. Mandibular plates and perichondrium enclosing the Meckel's cartilage showed strong expression of type I collagen in both wild type and gp 130-/- developing embryonic mandibles. The intramandibular Meckel's cartilage adjacent to the incisors (I) replaced by bone revealed type I collagen from E16.5 in wild type and from E17.5 in gp130-/- (original magnification $\times 40$ ). 

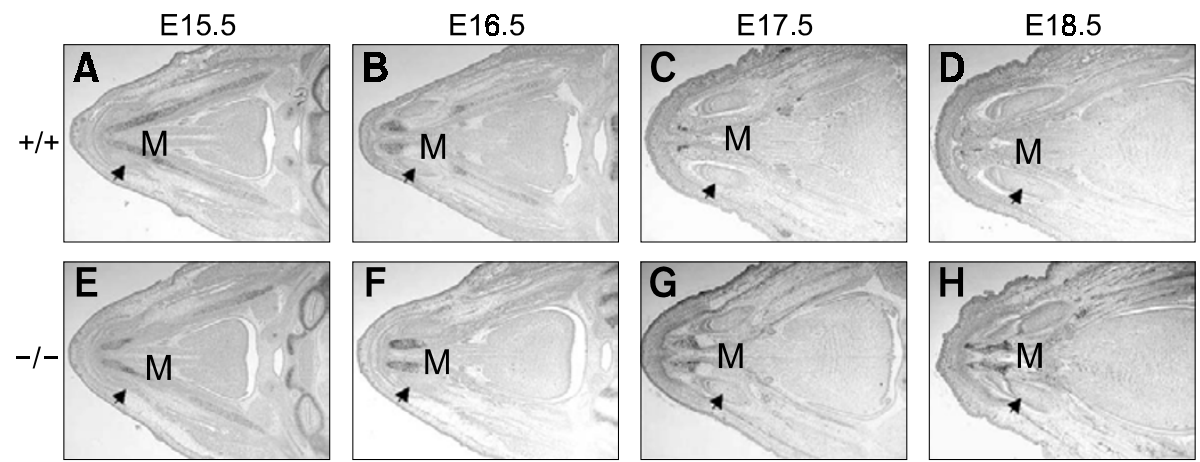

Figure 6. In situ hybridization for collagen type II mRNA in wild type (A-D) and gp130-/- (E-H) developing mandibles from E15.5 to E18.5. Type II collagen expression was onfined within Meckel's cartilage (M). However, this expression was gradually reduced in intramandibular and posterior portion of Meckel's cartilage, especially adjacent to the incisors (I) by hypertrophic maturation of chondrocytes from E16.5 in wild type and from E17.5 in gp130-/- (original magnification $\times 40$ ).

E15.5
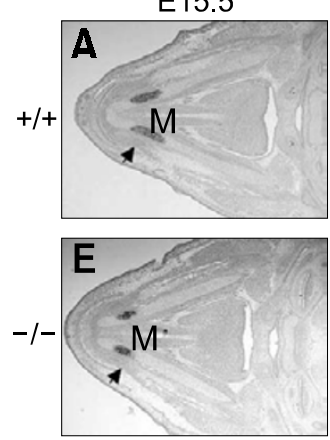

E16.5
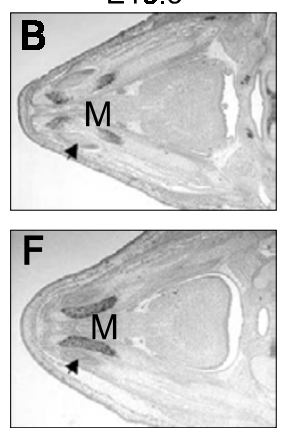

E17.5
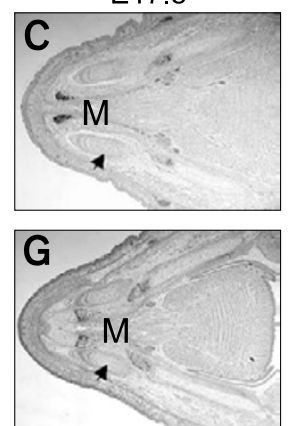

E18.5
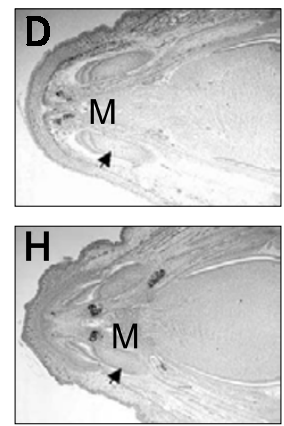

Figure 7. In situ hybridization for collagen type X mRNA in the wild type (A-D) and gp130-/- (E-H) developing mandible from E15.5 to E18.5. The expression of type $X$ collagen adjacent to the incisors (I) was eXtended in the anterior-posterior direction with time as the intramandibular Meckel's cartilage matured into hypertrophic chondrocytes. The zone of type X collagen expression was narrow in gp130-/- Meckel's cartilage (E) compared to that in the wild type (A). With replacement of Meckel's cartilage with bone, expression of type $\mathrm{X}$ collagen gradually disappeared, especially at adjacent to the incisors. This phenomenon progressed more rapidly in wild type (B-D) than in gp130-/- $(\mathrm{G}, \mathrm{H})$ (original magnification $\times 40)$.

Meckel's cartilage exhibited strong expression of type I collagen (Figure 5A-H). The intramendibular Meckel's cartilage which was replaced with bony tissue also expressed type I collagen gradually with time (Figure 5C, D, G, H). The type II collagen expression was observed throughout the Meckel's cartilage from the rostrum to the proximal ends (Figure 6A, E) except for the hypertrophic chondrocyte area adjacent to the developing incisors (Figure 6B-D, F-H). These areas revealed type $X$ collagen (Figure 7). The gp130-/- Meckel's cartilage also exhibited similar gene expression patterns but the type $X$ collagen-expressing zone was narrower than that observed in the wild type Meckel's cartilage (Figure 7D-H). With time, the type $X$ collagen expressing zone was extended to the anterior and posterior regions, with expression of type I collagen
(Figure 5C, D, G, H) instead of type $X$ collagen, suggesting bony replacement of the central portion of the hypertrophic chondrocyte area. This extension of the type $X$ collagen expressing zone in gp130-/- Meckel's cartilage was not as prominent as in the wild type Meckel's cartilage, and the central type I collagen expression was noted clearly from E17.5 (Figure 5G, H). The type II collagen expressing zone was gradually narrowed with thick type I collagen expressing lingual plate formation based on type X collagen expressing Meckel's cartilage. This transition of cartilage tissue into bone was more prominent in wild type Meckel's cartilage than in gp130-/- Meckel's cartilage (Figures 5-7). These findings suggest that gp130-mediated signaling does not disturb region specific expression of collagen type gene expression, but de- 
lays expression of the type $X$ collagen gene, as observed in retarded maturation of chondrocytes.

\section{Discussion}

Meckel's cartilage is regarded as the first skeletal component of the mandible (Macdonald and Hall, 2001). As in other developmental events, epithelial-mesenchymal interactions are required to initiate chondrogenesis of Meckel's cartilage and osteogenesis of the jaw bone. Meckel's cartilage is derived from cranial neural crest cells of the first branchial arch (Ishizeki et al., 2001). The proper development of Meckel's cartilage is critical for craniofacial skeletogenesis, and particularly for mandibular morphogenesis, because Meckel's cartilage serves as the primordium of the mandible and guides its early morphogenesis (Macdonald and Hall, 2001).

This study demonstrates that gp130-mediated signaling is important for the normal morphogenesis of Meckel's cartilage and subsequent mandibular development. gp130-deficient embryonic Meckel's cartilage exhibited characteristic bending of intramandibular portion where bony replacement occurs through the endochondral ossification mode. This bending of intramandibular Meckel's cartilage and associated mandibular bone resulted in a narrow mandibular arch, especially at the rostral area. The mandibular cortical bone plate formation associated with Meckel's cartilage was also poor. However, there was no specific change in collagen type gene expression, as determined by regional changes in chondrocyte characteristics.

gp130 is a non-ligand binding, signal-transducing $130-\mathrm{kD}$ glycoprotein consisting of 896 amino acids with a single transmembrane domain. It belongs to a class I cytokine receptor family (Taga and Kishimoto, 1997). Signaling via gp130 is initiated by the binding of cytokines with their cognate receptors, which induces homodimerization or heterodimerization of gp130 with LIFR or OSMR. This ligandinduced dimerization of gp130 triggers cytoplasmic signaling cascades by activating associated protein tyrosine kinases in the Janus kinase family (JAK1, JAK2, and TYK2). In addition to the JAK/STAT pathway, MAPKs might be involved in the membrane to nucleus signaling by IL- 6 related cytokines (Bravo and Heath, 2000; Mahboubi et al., 2003). gp130mediated signaling induces diverse biological effects in the immune, hematopoietic, and nervous systems and in bone homeostasis as well (Betz et al., 1998; Muller-Newen et al., 2000). Recently, gp130 was demonstrated to regulate bone turnover and bone size by at least two distinct intracellular pathways, STAT1/3 and SHP2/ras/MAPK, which are selectively activated in osteoblasts (Sims et al., 2004).

gp130-deficiency results in perinatal death from unknown causes (Yoshida et al., 1996) and causes shortened skeleton formation with bending of tubular bones, especially at the tibia (Shin et al., 2004). Bending of the tibia was noted in the gp130-/- tibia from the early stage at E16.5 when the bone collar is formed along the hypertrophied chondrocyte layers. The bone collar was thinner in the gp130-/embryonic tibia compared to that in the wild type tibia, and resorbed eccentrically with formation of a delta-shaped thick cortical palate at the center of diaphysis (Shin et al., 2004). The thin mineralized bone collar formation and eccentric resorption may cause unbalanced cortical bone strength, resulting in bending. This condition was also noted in developing gp130-/- embryonic mandibular growth. The ablation of gp130 gene revealed no remarkable difference in region-specific collagen mRNA expression between wild type and gp130-/- Meckel's cartilage and related osteogenic tissues but caused retardation of incremental growth of Meckel's cartilage and mandible with abnormal bending of intramandibular Meckel's cartilage where endochondral ossification has occurred. In situ hybridization studies revealed that type $X$ collagen was localized in the ossification region of intramandibular Meckel's cartilage, whereas type II collagen was found throughout the cartilage except for hypertrophied chondrocyte layers, which were replaced by bone (Ishizeki et al., 1998). The lateral side of the intramandibular Meckel's cartilage adjacent to the apex of the developing incisor expressing type $X$ collagen showed saucerized resorption by active osteoclasts and was ultimately integrated into the mandibular lingual plate through endochondral ossification. This transition of cartilage tissue into bone through endochondral ossification at hypertrophied chondrocyte zone was poor in gp130 deficient embryonic mice compared to wild type. This phenomenon was more prominent at E17.5 and E18.5. This delayed hypertrophic chondrocyte maturation and subsequent bony replacement caused characteristic bending of the intramandibular Meckel's cartilage and led subsequent bending of mandible. The bending might be resulted from inappropriate maturation of mineralized matrix of cartilage and replaced bone which could not allow enough resisting strength to pushing force of the developing incisors. Furthermore, the bending of mandible at transitional zone of Meckel's cartilage resulted in a narrow mandibular arch at the rostral area. This finding strongly suggests that the ablation of gp130 gene may cause no specific change in region-specific collagen mRNA expression but cause retardation of 
Meckel's cartilage and subsequent mandibular development with abnormal morphogenesis at intramandibulaur Meckel's cartilage undergoing bony replacement although the process is not known.

The transition pattern of hypertrophied chondrocyte zone into bone was unique in developing Meckel's cartilage. Unlike in the primary trabecular bone in the growth plate of the endochondral bone that was formed by apposition of bone matrix onto the resorbing cartilage core, the scaffolding activity was not observed in the remaining mineralized cartilage of intramandibular Meckel's cartilage where replacing of cartilage into bone is occurring. The gp130-/- embryos revealed significant retarded maturation of hypertrophic chondrocytes compared to wild type. The zone of expression of type $X$ collagen was narrower in gp130-/- Meckel's cartilage. The type $X$ collagen expressing area gradually expressed type I collagen, with loss of type $X$ collagen expression followed by replacement of mineralized cartilage by small trabecular bone intruding from outside of the Meckel's cartilage. The mineralized cartilage matrix was absorbed by TRAP-positive multinucleated giant cells which were characteristically larger and rounder with more nuclei than those in the wild type.

The differentiation of chondrocyte of Meckel's cartilage may be mainly regulated by genetic determinants but environmental influences may also concomitantly react (Chung and Nishimura, 1999; $\mathrm{Kim}$ et al., 2005). The developing incisor may be an environmentally affecting factor. The ablation of gp130 gene caused abnormal morphogenesis of Meckel's cartilage and subsequent mandible with retardation of incremental growth.

A variety of growth factors are important in embryonic Meckel's cartilage morphogenesis and associated mandibular development (Amano et al., 1999). The inhibition of EGFR expression (Shum et al., 1993; Miettinen et al., 1999) and inactivation of MMPs resulted in almost complete ablation of Meckel's cartilage (Ishizeki and Nawa, 2000). In addition, a critical role of the BMP-Msx-FGF signaling network has been reported in Meckel's cartilage and mandibular development (Macdonald and Hall, 2001; Mahboubi et al., 2003). TGF-mediated gene regulation also precisely controls embryonic organogenesis as well as Meckel's cartilage morphogenesis (Ito et al., 2002). Connective tissue growth factor was also recently reported to have an active role in Meckel's cartilage development (Shimo et al., 2004). PTHrP and Ihh constitute a signaling pathway that negatively regulates the rate of chondrocyte differentiation in endochondral bone formation (Long and Linsenmayer, 1998). The spatial and temporal pattern of $\mathrm{PTH}$ and $\mathrm{PTHrP}$ receptors localized in ossifying Meckel's cartilage is suggested to be closely related to the growth stage of Meckel's cartilage (Yamazaki et al., 1997). PTH, which shares a receptor with PTHrP, also plays a role in bone homeostasis by regulating gp130-associated cytokines such as IL-6, IL-11, and LIF (Taga and Kishimoto, 1997). The influence of gp130mediated signaling on Meckel's cartilage formation is not so critical as the effect of EGFR or MMPs. The delayed but relatively normal development of Meckel's cartilage formation at early stage suggests that gp130-mediated signaling is more important in later stage of Meckel's cartilage development, especially at bony replacement stage and associated mandilbular plate development stage. This finding also indicates that gp130-mediated signaling is more influencing on osteogenesis than chondrogenesis. Recently, it has been demonstrated that the gp130-mediated signaling is necessary for not only normal osteoblastic function but also osteoclastic differentiation and activation (Shin et al., 2004). The deficiency of gp130-mediedated signaling caused poor osteoblastic differentiation and mineral nodule formation in vitro and shortening of bone with reduced bone volume in vivo (Shin et al., 2004; Sims et al., 2004).

Growth retardation of the mandible may lead to disturbed palate formation; for example, abrogation of the chondrodysplasia gene causes cleft palate due to retarded mandibular development and interference from forward displacement of the tongue (Seegmiller and Fraser, 1997). However, there was no notable disturbance in palatal development in this study.

In conclusion, the present study demonstrates that ablation of the gp130 gene causes shortened embryonic Meckel's cartilage, delayed hypertrophic maturation of chondrocytes and subsequent bony replacement with characteristic bending of the intramandibular Meckel's cartilage. Furthermore, the bent Meckel's cartilage integrated into the mandibular lingual plate led to a narrow mandibular arch at the rostral area. Taken together, these findings indicate that gp130-mediated signaling is important for proper mandibular morphogenesis and development.

\section{Acknowledgment}

This study was supported by grant of the Korea Health 21 R\&D project, Ministry of Health and Welfare (01-PJ3PG6-01GN11-0002) and grant (M10646020001-06N460200110) from the national R\&D Program of MOST and KOSEF. 


\section{References}

Amano O, Koshimizu U, Nakamura T, Iseki S. Enhancement by hepatocyte growth factor of bone and cartilage formation during embryonic mouse mandibular development in vitro. Archives of Oral Biology 1999;44:935-46

Betz UA, Bloch W, Brock M, Yoshida K, Taga T, Kishmoto T, Addicks K, Rajewsky K, Muller W. Postnatally induced inactivation of gp130 in mice results in Neurological, cardiac, hematopoietic, immunological hepatic and pulmonary defects. J Exp Med 1998;188:1955-65

Bravo J, Heath JK. Receptor recognition by gp130 cytokines. EMBO J 2000;19:2399-411

Chung KS, Nishimura I. Maintenance of regional histodifferentiation patterns and a spatially restricted expression of type $X$ collagen in rat Meckel's cartilage explants in vitro. Archives of Oral Biology 1999;44:489-97

Harada Y, Ishizeki K. Evidence for transformation of chondrocytes and site-specific resorption during the degradation of Meckel's cartilage. Anat Embryol 1998;197:439-50

Hetnabb D, Rousselle A. Gp130 cytokine family and bone cells. Cytokine 2000;12:1455-68

Ishizeki K, Kubo M, Yamamoto H, Nawa T. Immunocytochemical expression of type I and type II collagens by rat Meckel's cartilage chondrocytes in culture during phenotypic transformation. Archives of Oral Biology 1998;43:117-26

Ishizeki K, Nawa T. Further evidence for secretion of matrix metalloproteinase- 1 by Meckel's chondrocytes during degradation of the extracellular matrix. Tissue and Cell 2000;32: 207-15.

Ishizeki K, Takahashi N, Nawa T. Formation of the sphenomandibular ligament by Meckel's cartilage in the mouse: possible involvement of epidermal growth factor as revealed by studies in vivo and in vitro. Cell Tissue Res 2001;304:67-80

Ito $\mathrm{Y}$, Bringas PJ, Mocharei A, Zhao J. Receptor-regulated and inhibitory Smads are critical in regulating transforming growth factor $\beta$-mediated Meckel's cartilage development. Developmental Dynamics 2002;224:69-78

Kim JH, Do HJ, Yang HM, Oh JH, Choi SJ, Kim DK, Cha $\mathrm{KY}$, Chung HM. Overexpression of SOX9 in mouse embryonic stem cells directs the immediate chondrogenic commitment. Exp Mol Med 2005;37:261-8

Lanske B, Amling M, Neff L, Guiducci J, Baron R, Kronenberg $\mathrm{HM}$. Ablation of the PTHrP gene or the PTH/PTHrP receptor gene leads to distinct abnormalities in bone development. $J$ Clin Invest 1999;104:399-407

Long F, Linsenmayer TF. Regulation of growth region cartilage proliferation and differentiation by perichondrium. Development 1998;125:1067-73

Macdonald ME, Hall BK. Altered timing of the extracellular-matrix-mediated epithelial-mesenchymal interaction that initiates mandibular skeletogenesis in three inbred strains of mice: development, heterochrony, and evolutionary change in morphology. J Exp Zool 2001;291:258-73

Mahboubi K, Kirkiles-Smith NC, Karras J, Pober JS. Desensitization of signaling by oncostatin $\mathrm{m}$ in human vascular cells involves cytoplasmic Tyr residue 759 in gp130 but is not mediated by either Src homology 2 domain-containing tyrosine phosphatase 2 or suppressor of cytokine signaling 3 . J Biol Chem 2003;278:25014-23

Miettinen PJ, Chin JR, Shum L, Slavkin HC, Shuler CF,
Derynck R, Werb Z. Epidermal growth factor receptor function is necessary for normal craniofacial development and palate closure. Nature Genetics 1999;22:69-73

Miyake T, Cameron AM, Hall BK. Stage-specific onset of condensation and matrix deposition for Meckel's and other first arch cartilages in inbred C57BL/6 mice. J Craniofac Genet Dev Biol 1996;16:32-47

Muller-Newen G, Kuster A, Wijdenes J, Schaper F, Heinrich PC. Studies on the interleukin-6-type cytokine signal transducer gp130 reveal a novel mechanism of receptor activation by monoclonal antibodies. J Biol Chem 2000;257:4579-86

Nicole M, Kai W, Christa B, Fabio Q, Wolfgang S, Rainer H. Light and electronmicroscopic in-situ hybridization of collagen type I and type II mRNA in the fibrocartilaginous tissue of late-stage osteoarthritis. Osteoarthritis and Cartilage 1998; 6:278-85

Nifuji A, Kellermann O, Kuboki Y, Wozney JM, Noda M. Perturbation of BMP signaling in somatogenesis resulted in vertebral and rib malformations in the axial skeletal formation. J Bone Miner Res 1997;12:332-42

Seegmiller RE, Fraser FC. Mandiblular growthretardation as a cause of cleft plate in mice homozygous for the chondrodyspalsia gene. J Embryol Exp Morphol 1997;38:227-38

Shimo T, Kanyama M, Wu C, Sugito $H$, Billings PC, Abrams WR, Rosenbloom J, Lwamoto M, Pacifici M, Koyama E. Expression and roles of connective tissue growth factor in Meckel's cartilage development. Developmental Dynamics 2004;231:136-47

Shin HI, Divieti P, Sims NA, Kobayashi T, Miao D, Karaplis AC, Baron R, Bringhurst R, Kronenberg HM. Gp130-mediated signaling is necessary for normal osteoblastic function in vivo and in vitro. Endocrinology 2004;145:1376-85

Shum L, Sakakura Y, Bringas P, Wen LJ, Snead ML, Mayo $M$, Crohin C, Millar S, Werb Z, Buckley S, Hall FL, Warburton $D$, Slavkin HC. EGF abrogation-induced fusilli-form dysmorphogenesis of Meckel's cartilage curing embryonic mouse mandibular morphogenesis in vitro. Development 1993;118: 903-17

Sims NA, Jenkins BJ, Quinn JM, Nakamura A, Glatt M, Gillespie MT, Ernst M, Martin TJ. Glycoprotein 130 regulates bone turnover and bone size by distinct downstream signaling pathway. J Clin Invest 2004;113:379-89

Taga T, Kishimoto T. Gp130 and the interleukin- 6 family of cytokines. Annu Rev Immunol 1997;15:979-819

Tomo S, Ogita M, Tomo I. Development of mandibular cartilages in the rat. Anat Rec 1997;249:233-9

Wilson J, Tucker AS. Fgf and Bmp signals repress the expression of Bapx1 in the mandibular mesenchyme and control the position of the developing jaw joint. Developmental Biology 2004;266:138-50

Yamazaki K, Suda N, Kuroda T. Immunohistochemical localization of parathyroid hormone-related protein in developing mouse Meckel's cartilage and mandible. Archs oral Biol 1997;42:787-94

Yoshida K, Taga T, Saito M, Suematsu S, Kumanogoh A, Tanaka T, Fujiwara H, Hirata M, Yamagami T, Nakahata T, Hirabayashi T, Yoneda Y, Tanaka K, Wang WZ, Mori C, Shiota K, Yoshida N, Kishimoto T. Targeted disruption of gp130, a common signal transducer for the interleukin 6 family of cytokines, leads to myocardial and hematological disorders. Proc Natl Acad Sci USA 1996;93:407-11 